\title{
Renal functions in obstructive sleep apnea patients
}

\author{
${\text { Meral } \operatorname{Uyar}^{1}}^{(1)}$
}

Received: 19 January 2017 / Accepted: 26 January 2017 / Published online: 3 February 2017

(C) Springer-Verlag Berlin Heidelberg 2017

Dear Editor,

We have read Kawada's views on our study with interest. There are a few points to clarify:

Namely, our study was a case-control study evaluating kidney function and confounding parameters in patients with obstructive sleep apnea (OSA). Patients with chronic kidney disease were excluded. Serum creatinin level was significantly higher among patients with OSA and in the nominal regression model that was utilized, and diabetes was the only independent variable after adjustment for other confounders.

In our study, the rate of metabolic syndrome (MetS) was not different between the control and patient groups; however, among OSA patients, eGFR was significantly lower in patients with MetS. Therefore, we have suggested that coexistence of OSA and MetS may contribute to renal impairment. Further studies evaluating causal associations are needed as Kawada suggested and may have an impact on everyday practice.

Ogna et al. [1] studied sleep characteristics in patients with early-stage chronic kidney disease which examine the association of kidney function and sleep. OSA diagnosis was based on home sleep recordings, and only patients with moderate and severe OSA were included in data analysis. Major difference between the two studies is in the selection of the study population. We have included patients with every stage of sleep apnea without chronic kidney disease which presents a different perspective.

Finally, regarding the effects of OSA specific therapy on renal function, there are a number of studies showing positive impact [2,3]. All of our analyses were done on treatment naive patients. The effect of treatment on renal functions in patients with sleep disordered breathing is a broad study area.

\section{References}

1. Ogna A, Forni Ogna V, Haba Rubio J et al (2016) Sleep characteristics in early stages of chronic kidney disease in the HypnoLaus cohort. Sleep 39(4):945-953

2. Chen LD, Lin L, Ou YW et al (2016) Effect of positive airway pressure on glomerular filtration rate in patients with sleepdisordered breathing: a meta-analysis. Sleep Breath. doi:10.1007/ s11325-016-1364-6

3. Nicholl DD, Hanly PJ, Poulin MJ et al (2014) Evaluation of continuous positive airway pressure therapy on renin-angiotensin system activity in obstructive sleep apnea. Am J Respir Crit Care Med 190(5):572-580. doi:10.1164/rccm.201403-0526OC
Meral Uyar

meraluyar1@yahoo.com

1 Department of Respiratory Medicine, Faculty of Medicine, Gaziantep University, Gaziantep, Turkey 